\title{
Quintic B-Spline Technique for Numerical Treatment of Third Order Singular Perturbed Delay Differential Equation
}

\author{
Mandeep Kaur Vaid \\ Department of Mathematics, \\ Lovely Professional University, Phagwara, Punjab, India \\ Geeta Arora \\ Department of Mathematics, \\ Lovely Professional University, Phagwara, Punjab, India \\ Corresponding author: geetadma@gmail.com
}

(Received April 14, 2019; Accepted August 7, 2019)

\begin{abstract}
In this paper, a class of third order singularly perturbed delay differential equation with large delay is considered for numerical treatment. The considered equation has discontinuous convection-diffusion coefficient and source term. A quintic trigonometric B-spline collocation technique is used for numerical simulation of the considered singularly perturbed delay differential equation by dividing the domain into the uniform mesh. Further, uniform convergence of the solution is discussed by using the concept of Hall error estimation and the method is found to be of first-order convergent. The existence of the solution is also established. Computation work is carried out to validate the theoretical results.
\end{abstract}

Keywords- Quintic trigonometric spline, Error estimate, Perturbed equation, Delay.

\section{Introduction}

The frequent emerge of singular perturbed delay differential equations (SPDDE) in every field of science and technology has triggered the researchers for numerical treatment of these equations. These equations involve two sensitive parameters: perturbation $(\boldsymbol{\varepsilon})$ and retarded (or delay) $(\boldsymbol{\delta})$ parameter. The study of these differential equations is a stiff job for the researchers due to startlingadapt of the solution at boundary as $\boldsymbol{\varepsilon} \rightarrow \mathbf{0}$, and such variation in solution at the boundary is well known as boundary layer. The other factor which provokes the mathematicians for analysis of SPDDE is recurrent emerge of these equations in real life problems. Ample mathematical models in science and technology result in SPDDE. Reader can refer (Stein, 1965; Stein, 1967; Longtin and Milton, 1988; Nelson and Perelson, 2002; Rihan, 2013; Wilkie and Hahnfeldt, 2013) for some of applications of SPDDE in biosciences.

The delay differential equations are also the result of mathematical models of the real-life problems in various fields. One such model exists in study of the development of the bearing of the population of a system of organisms. This system can be well explained by its mathematical modeling with delay as the prominent insight of the dynamic conduct of the system. This time delay appears in a polluted ecosystem, and is one of the important factors affecting the expansion of the biological system. Therefore, delay differential equations are the result of mathematical modeling explaining organic structure. The disparity and bifurcation in the system emerge due to the time delay. One of the common bifurcations (the division of system into two parts) is hop bifurcation which refers to the division of the system at a point where the firmness turns around 
International Journal of Mathematical, Engineering and Management Sciences

Vol. 4, No. 6, 1471-1482, 2019

https://dx.doi.org/10.33889/IJMEMS.2019.4.6-116

and periodic solution arises as a particular variable changes its values. One such mathematical model of the singular biological system is proposed by Zhang et al. (2016) by considering the delay parameter into account.

The model is framed by considering $\tau$ as the time delay of the transformation of immature organisms into mature organisms, $\mathrm{u}(\mathrm{t})$ and $\mathrm{v}(\mathrm{t})$ taken as the densities of the immature and mature organisms, $E(t)$ as capture capability of mature creatures at time $t, p_{1}$ as a unit price, $c$ is a unit cost, and $m$ is economic profit. $p_{1} v(t) E(t)$ represents the total revenue, and $c E(t)$ denotes the total cost. The delay model of the biological system given by:

$$
\begin{aligned}
& \dot{u}(t)=p v(t)-q u(t-\tau)-d_{1} u(t)-\phi_{1} u_{1}(t) u(t) \\
& \dot{v}(t)=q u(t-\tau)-d_{2} v(t)-\beta v^{2}(t)-E(t) v(t)-\phi_{2} u_{1}(t) v(t) \\
& \text { and } \dot{u}_{1}(t)=\theta-h u_{1}(t), 0=E(t)\left(p_{1} v(t)-c\right)-m
\end{aligned}
$$

A range of numerical methods have been proposed in recent years for numerical treatment of these differential equations. Some of the reported work can be cited from (Andargie and Reddy, 2013; Nicaise and Xenophontos, 2013; Swamy et al., 2015; Cimen, 2017). With the incitement from the reported work, this paper is an extension of numerical simulation of SPDDE.

This paper is organized as follows. In the next section (Section 2) the considered SPDDE is stated followed by the existence of the solution of the problem stated in Section 3. In Section 4, the method applied is interpreted with convergence of the scheme explored in Section 5. Section 6, involves the estimated solution for some numerical examples with conclusion in Section 7.

\section{Problem Statement}

The problem is to find solution $y \epsilon C^{1}(\bar{\Omega}) \cap C^{2}(\Omega) \cap C^{3}\left(\Omega^{*}\right)$ so that it satisfies the following equation:

$$
\begin{gathered}
L y(x)=-\varepsilon y^{\prime \prime \prime}(x)+a(x) y^{\prime \prime}(x)+b(x) y^{\prime}(x)+c(x) y(x)+d(x) y^{\prime}(x-1) \\
=f(x), x \in \Omega^{*}
\end{gathered}
$$

with boundary conditions

$$
y(x)=\phi(x), x \in[-1,0], y^{\prime}(2)=\gamma
$$

where $\varepsilon$ is very-very small positive number and $a(x), f(x)$ are discontinuous functions as shown below:

$a(x)=\left\{\begin{array}{l}a_{1}(x), x \in[0,1] \\ a_{2}(x), x \in(1,2]\end{array}\right.$ and $f(x)=\left\{\begin{array}{l}f_{1}(x), x \in[0,1] \\ f_{2}(x), x \in(1,2]\end{array}\right.$

with the conditions $a_{1}(1-) \neq a_{2}(1+), f_{1}(1-) \neq f_{2}(1+), a_{i}(x)>\alpha_{i}(x)>\alpha+2>3$, $b(x) \geq \beta_{0} \geq 0, \gamma_{0} \leq c(x) \leq \gamma \leq 0, \eta_{0} \leq d(x) \leq 0$ such that $2 \alpha+4 \gamma_{0}+5 \eta_{0}>0$ (Subburayan and Mahendran, 2018). 
International Journal of Mathematical, Engineering and Management Sciences

Vol. 4, No. 6, 1471-1482, 2019

https://dx.doi.org/10.33889/IJMEMS.2019.4.6-116

Here, the functions $a(x)$ and $f(x)$ are sufficiently smooth and bounded on $\Omega^{*}, b(x), c(x)$ and $d(x)$ are sufficiently smooth functions on $\bar{\Omega}$, where $\Omega^{*}=\Omega^{+} \cup \Omega^{-}, \Omega^{-}=(0,1), \Omega^{+}=(1,2)$ and $\Omega=$ $(0,2)$.

Now, to handle the delay parameter Taylor's series up to second order accuracy is used as

$$
y^{\prime}(x-1)=y^{\prime}(x)-y^{\prime \prime}(x)
$$

By using Taylor's series expansion in equation (1), we get the equation in form:

$P(x) y^{\prime \prime \prime}(x)+Q(x) y^{\prime \prime}(x)+R(x) y^{\prime}(x)+S(x) y(x)=f(x)$

where $P(x)=-\varepsilon, Q(x)=a(x)-d(x), R(x)=b(x)+d(x)$ and $S(x)=c(x)$.

\section{Existence of Solution}

Theorem: The equation (1)-(2) has solution $\bar{y}=\left(y_{1}, y_{2}\right)$ where $y_{1} \epsilon C^{0}(\bar{\Omega}) \cap C^{1}(\Omega \cup\{2\})$ and $y_{2} \in C^{0}(\bar{\Omega}) \cap C^{1}(\Omega) \cap C^{2}\left(\Omega^{*}\right)$.

Proof: Proof of this theorem is by construction.

Let $\overline{y_{L}}=\left(y_{L A}, y_{L B}\right)$ and $\overline{y_{R}}=\left(y_{R A}, y_{R B}\right)$ be the particular solution of the following problems:

$$
\left\{\begin{array}{c}
y_{L A}^{\prime}(x)-y_{L B}(x)=0, x \in \Omega^{-} \\
-\varepsilon y_{L B}^{\prime \prime \prime}(x)+a(x) y_{L B}^{\prime \prime}(x)+b(x) y_{L B}^{\prime}(x)+c(x) y_{L A}(x)+d(x) y_{L A}^{\prime}(x-1)=f(x), x \in \Omega^{-} \\
y_{L A}(x)=\phi(x), y_{L B}=\phi^{\prime}(x), x \in[-1,0]
\end{array}\right.
$$

and

$$
\left\{\begin{array}{c}
y_{R A}^{\prime}(x)-y_{R B}(x)=0, x \in \Omega^{+} \\
-\varepsilon y_{R B}^{\prime \prime \prime}(x)+a(x) y_{R B}^{\prime \prime}(x)+b(x) y_{R B}^{\prime}(x)+c(x) y_{R A}(x)+d(x) y_{R A}^{\prime}(x-1)=f(x), x \in \Omega^{+}
\end{array}\right.
$$

Now we consider the function $\bar{y}=\left(y_{1}, y_{2}\right)$ as:

$$
\begin{aligned}
& y_{1}(x)=\left\{\begin{array}{c}
y_{L A}(x)+A \emptyset_{1 A}(x), x \in \Omega^{-} \\
y_{R A}(x)+\emptyset_{2 A}(x)\left[y_{2}(2)-y_{R A}(2)\right]+B \emptyset_{3 A}(x), x \in \Omega^{+}
\end{array}\right. \\
& y_{2}(x)=\left\{\begin{array}{c}
y_{L B}(x)+A \emptyset_{1 B}(x), x \in \Omega^{-} \\
y_{R B}(x)+\emptyset_{2 B}(x)\left[y_{2}(2)-y_{R B}(2)\right]+B \emptyset_{3 B}(x), x \in \Omega^{+}
\end{array}\right.
\end{aligned}
$$

where the functions $\overline{\emptyset_{1}}=\left(\varnothing_{1 A}, \emptyset_{1 B}\right), \overline{\emptyset_{2}}=\left(\varnothing_{2 A}, \emptyset_{2 B}\right)$ and $\overline{\emptyset_{3}}=\left(\emptyset_{3 A}, \emptyset_{3 B}\right)$ satisfies the following boundary value problems respectively:

$$
\left\{\begin{array}{c}
\emptyset_{1 A}^{\prime}(x)-\emptyset_{1 B}(x)=0, x \in \Omega \bigcup\{2\} \\
-\varepsilon \emptyset_{1 B}^{\prime \prime \prime}(x)+a(x) \emptyset_{1 B}^{\prime \prime}(x)+b(x) \emptyset_{1 B}^{\prime}(x)+c(x) \emptyset_{1 A}(x)+d(x) \emptyset_{1 A}^{\prime}(x-1)=f(x), x \in \Omega \\
\emptyset_{1 A}(x)=0 \text { for } x[-1,0], \emptyset_{1 B}(x)=0 \text { for } \epsilon[-1,0] \text { and } \emptyset_{1 B}(2)=1
\end{array}\right.
$$


International Journal of Mathematical, Engineering and Management Sciences

Vol. 4, No. 6, 1471-1482, 2019

https://dx.doi.org/10.33889/IJMEMS.2019.4.6-116

$$
\begin{gathered}
\left\{\begin{array}{c}
\emptyset_{2 A}^{\prime}(x)-\emptyset_{2 B}(x)=0, x \in \Omega \cup\{2\} \\
-\varepsilon \emptyset_{2 B}^{\prime \prime \prime}(x)+a(x) \emptyset_{2 B}^{\prime \prime}(x)+b(x) \emptyset_{2 B}^{\prime}(x)+c(x) \emptyset_{2 A}(x)+d(x) \emptyset_{2 A}^{\prime}(x-1)=f(x), x \in \Omega \\
\emptyset_{2 A}(x)=0 \text { for } \epsilon[-1,0], \emptyset_{2 B}(x)=0 \text { for } \epsilon[-1,0] \text { and } \emptyset_{2 B}(2)=1
\end{array}\right. \\
\left\{\begin{array}{c}
\emptyset_{3 A}^{\prime}(x)-\emptyset_{3 B}(x)=0, x \in \Omega U\{2\} \\
-\varepsilon \emptyset_{3 B}^{\prime \prime \prime}(x)+a(x) \emptyset_{3 B}^{\prime \prime}(x)+b(x) \emptyset_{3 B}^{\prime}(x)+c(x) \emptyset_{3 A}(x)+d(x) \emptyset_{3 A}^{\prime}(x-1)=f(x), x \in \Omega \\
\emptyset_{3 A}(x)=0 \text { for } x[-1,0], \emptyset_{3 B}(x)=0 \text { for } x \in[-1,0] \text { and } \emptyset_{3 B}(2)=0 .
\end{array}\right.
\end{gathered}
$$

It is apparent that $\overline{\boldsymbol{y}}$ satisfy (1)-(2) and the constants A and B can be obtained by procedure given by Subburayan and Mahendran (2018) to show existence of the solution.

\section{Quintic Trigonometric B-Spline Collocation Method}

The first order trigonometric function is given by

$$
T S_{1, i}(x)=\left\{\begin{array}{lr}
1, \text { for } & x_{i} \leq x<x_{i+1} \\
0, & \text { otherwise }
\end{array}\right.
$$

and the $\tau^{\text {th }}$ ordered trigonometric $\mathrm{i}^{\text {th }}$ function is given by

$$
T S_{\tau, i}(x)=\sin \left(\frac{x-x_{i}}{2}\right) B_{\tau-1, i}(x)+\sin \left(\frac{x_{i+\tau}-x}{2}\right) B_{\tau-1, i+1}(x)
$$

where $B_{\tau-1, \gamma}(x)=\left\{\begin{array}{c}\frac{T_{\tau-1, \gamma}(x)}{\sin \left(\frac{x_{\gamma+\tau+1}-x_{\gamma}}{2}\right)} \\ 0, x_{\gamma}=x_{\gamma+\tau-1}\end{array}, x_{\gamma}<x_{\gamma+\tau-1}\right.$ for $\gamma=i$ or $\gamma=i+1$.

The quintic trigonometric $b$-spline collocation method is used for the numerical simulation of the considered SPDDE. The domain $0 \leq x \leq 2$ is partitioned into a uniform mesh with each sub intervals of length $1 / N$ with resultant partition $\left\{0=x_{0}<x_{1}<x_{2}<\ldots \ldots . x_{N}=2\right\}$ where $N$ is the total number of partition points and $\left\{T_{5,-2}(x), T_{5,-1}(x), \ldots, T_{5, N+2}(x)\right\}$ form the basis for function defined over the interval $\left[x_{0}, x_{N}\right]$.The approximated solution is considered as:

$y(x)=\sum_{i=-2}^{N+2} \alpha_{i} T_{5, i}(x)$

where $T_{5, i}(x)$ 's are the trigonometric B-spline basis function of fifth order as described by Zakaria et al. (2017) and $\alpha_{i}$ 's are unknown coefficients to be determined. At the nodal points $x_{i}$, the function $\mathrm{y}(\mathrm{x})$ and its derivatives are defined as:

$$
\begin{aligned}
& y\left(x_{i}\right)=k_{1} \alpha_{i-2}+k_{2} \alpha_{i-1}+k_{3} \alpha_{i}+k_{2} \alpha_{i+1}+k_{1} \alpha_{i+2}, y^{\prime}\left(x_{i}\right)=k_{4} \alpha_{i-2}+k_{5} \alpha_{i-1}+k_{6} \alpha_{i+1}+k_{7} \alpha_{i+2} \\
& y^{\prime \prime}\left(x_{i}\right)=k_{8} \alpha_{i-2}+k_{9} \alpha_{i-1}+k_{10} \alpha_{i}+k_{9} \alpha_{i+1}+k_{8} \alpha_{i+2} \quad, \quad y^{\prime \prime \prime}\left(x_{i}\right)=k_{11} \alpha_{i-2}+ \\
& k_{12} \alpha_{i-1}+k_{13} \alpha_{i+1}+k_{14} \alpha_{i+2}
\end{aligned}
$$

where the constants $k_{i}$ for quintic Trigonometric B-spline are as follows:

$$
k_{1}=\frac{\sin (h / 4)^{4}}{\sin h \sin \left(\frac{3 h}{2}\right) \sin (2 h) \sin \left(\frac{5 h}{2}\right)}, k_{2}=\frac{5+8 \cos h}{4 \cosh \cos \left(\frac{h}{2}\right)(1+2 \cos h)(1+2 \cos h+2 \cos 2 h)},
$$


International Journal of Mathematical, Engineering and Management Sciences

Vol. 4, No. 6, 1471-1482, 2019

https://dx.doi.org/10.33889/IJMEMS.2019.4.6-116

$$
\begin{aligned}
& k_{3}=\frac{6+6 \cosh \operatorname{sech} \sec (\mathrm{h} / 2)^{2}}{4+8 \cos h+8 \cos 2 h}, k_{4}=\frac{-5 \sin (h / 2)^{2}}{4 \sin (3 h / 2) \sin (2 h) \sin (5 h / 2)}, k_{5}=\frac{-5(1+4 \cos h) \operatorname{cosec}(h / 2) \sec (h)}{8(1+2 \cos h)(1+2 \cos h+2 \cos 2 h)}, \\
& k_{6}=\frac{5(1+4 \cos h) \operatorname{cosec}(h / 2) \sec (h)}{8(1+2 \cos h)(1+2 \cos h+2 \cos 2 h)}, k_{7}=\frac{5 \sin (h / 2)^{2}}{4 \sin (3 h / 2) \sin (2 h) \sin (5 h / 2)}, \\
& k_{8}=\frac{5(3+5 \cos h)}{16 \cosh (1+2 \cos h)(1+2 \cos h+2 \cos 2 h) \sin (h)^{2}}, \\
& k_{9}=\frac{5(3+5 \cos h)}{32 \cosh \cos (h / 2)(1+2 \cos h)(1+2 \cos h+2 \cos 2 h) \sin (h / 2)^{2}}, k_{10}=\frac{5(2+5 \cos h+2 \cos 2 h)}{8 \cosh (1+2 \cos h+2 \cos 2 h) \sin (h)^{2}}, \\
& k_{11}=\frac{-5(-1+25 \cos h)}{128 \cosh \cos (h / 2)(1+2 \cos h)(1+2 \cos h+2 \cos 2 h) \sin (h / 2)^{3}}, \\
& k_{12}=\frac{-5(1-27 \cos h+2 \cos 2 h)}{64 \cosh (1+2 \cos h)(1+2 \cos h+2 \cos 2 h) \sin (h / 2)^{3}}, \\
& k_{13}=\frac{5(1-27 \cos h+2 \cos 2 h)}{64 \cosh (1+2 \cos h)(1+2 \cos h+2 \cos 2 h) \sin (h / 2)^{3}}, \\
& k_{14}=\frac{5(-1+25 \cos h)}{128 \cos h \cos (h / 2)(1+2 \cos h)(1+2 \cos h+2 \cos 2 h) \sin (h / 2)^{3}} .
\end{aligned}
$$

Now, to apply the collocation technique collocation points are selected in such a way that they concur with the nodal points. By substituting the values of $y_{i}, y_{i}^{\prime}, y_{i}{ }^{\prime \prime} a n d y_{i}{ }^{\prime \prime \prime}$ at the nodal points in equation (3) we get a system of $N+1$ linear equations in $N+5$ unspecified variables as:

$W_{1}^{i} \alpha_{i-2}+W_{2}{ }^{i} \alpha_{i-1}+W_{3}{ }^{i} \alpha_{i}+W_{4}{ }^{i} \alpha_{i+1}+W_{5}{ }^{i} \alpha_{i+2}=f_{i}, \quad 0 \leq i \leq N$

where $W_{1}^{i}=k_{11} P(x)+k_{8} Q(x)+k_{4} R(x)+k_{1} S(x)$,

$$
\begin{aligned}
& W_{2}^{i}=k_{12} P(x)+k_{9} Q(x)+k_{5} R(x)+k_{2} S(x), W_{3}^{i}=k_{10} Q(x)+k_{3} S(x) \\
& W_{4}^{i}=k_{13} P(x)+k_{9} Q(x)+k_{6} R(x)+k_{2} S(x), W_{5}{ }^{i}=k_{14} P(x)+k_{8} Q(x)+k_{7} R(x)+k_{1} S(x)
\end{aligned}
$$

To calculate the four unknown variables $\alpha_{-1}, \alpha_{-2}, \alpha_{N+1}$ and $\alpha_{N+2}$, one can use the two given boundary conditions and two assumed conditions as following:

(i) $y_{0}=\varnothing$ (ii) $y^{\prime}(2)=\gamma$ and (iii) $y^{\prime \prime}(0)=0$, (iv) $y^{\prime \prime}(2)=0$.

By solving the system of equations $y_{0}=\emptyset$ and $y^{\prime \prime}(0)=0$, we obtained values of $\alpha_{-2}$ and $\alpha_{-1}$ as:

$\alpha_{-2}=\frac{L k_{2}-R k_{9}}{k_{8} k_{2}-k_{1} k_{9}}=A_{1}($ say $)$ and $\alpha_{-1}=\frac{R k_{8}-L k_{1}}{k_{8} k_{2}-k_{1} k_{9}}=A_{2}$

where, $\mathrm{L}=-k_{10} \alpha_{0}-k_{9} \alpha_{1}-k_{8} \alpha_{2}$ and $\mathrm{R}=\emptyset-k_{3} \alpha_{0}-k_{2} \alpha_{1}-k_{1} \alpha_{2}$.

Similarly, by solving the system of equations $y^{\prime}(2)=\gamma$ and $y^{\prime \prime}(2)=0$, we obtained values of $\alpha_{N+2}$ and $\alpha_{N+1}$ as:

$\alpha_{N+1}=\frac{L_{1} k_{7}-R_{1} k_{8}}{k_{9} k_{7}-k_{8} k_{6}}=A_{3}$ and $\alpha_{N+2}=\frac{R_{1} k_{9}-L_{1} k_{6}}{k_{9} k_{7}-k_{8} k_{6}}=A_{4}$ 
International Journal of Mathematical, Engineering and Management Sciences

Vol. 4, No. 6, 1471-1482, 2019

https://dx.doi.org/10.33889/IJMEMS.2019.4.6-116

where, $L_{1}=-k_{8} \alpha_{N-2}-k_{9} \alpha_{N-1}-k_{10} \alpha_{N}$ and $R_{1}=\gamma-k_{4} \alpha_{N-2}-k_{5} \alpha_{N-1}$.

Substituting these values in equation (5) for $i=0,1, N-1$ and $i=N$, we get following equations:

$\alpha_{0} W_{3}^{0}+\alpha_{1} W_{4}{ }^{0}+\alpha_{2} W_{5}{ }^{0}=f_{0}-A_{1} W_{1}{ }^{0}-A_{2} W_{2}{ }^{0}$

$\alpha_{0} W_{2}^{1}+\alpha_{1} W_{3}^{1}+\alpha_{2} W_{4}^{1}+\alpha_{3} W_{5}^{1}=f_{1}-A_{2} W_{1}^{1}$

$\alpha_{N-3} W_{1}^{N-1}+\alpha_{N-2} W_{2}^{N-1}+\alpha_{N-1} W_{3}^{N-1}+\alpha_{N} W_{4}^{N-1}=f_{N-1}-A_{3} W_{5}^{N-1}$

and

$\alpha_{N-2} W_{1}^{N}+\alpha_{N-1} W_{2}^{N}+\alpha_{N} W_{3}^{N}=f_{N}-A_{3} W_{4}^{N}-A_{4} W_{5}^{N}$

Now, by considering above equations (6) to (9) and other equations from equation (5) for $\mathrm{i}=2,3$ ,.., $N-2$, a system of order $N+1$ is obtained with $N+1$ variables as A $\alpha=\mathrm{B}$ where $\alpha=$ $\alpha_{0}, \alpha_{1}, \alpha_{2}, \ldots \ldots \ldots, \alpha_{N}$. A is a penta-diagonal matrix given by

$$
\mathrm{A}=\left[\begin{array}{cccccccc}
W_{3}^{0} & W_{4}^{0} & W_{5}^{0} & \cdots & \cdots & \cdots & \cdots & 0 \\
W_{2}^{1} & W_{3}^{1} & W_{4}^{1} & W_{5}^{1} & \cdots & \cdots & \cdots & 0 \\
W_{1}^{2} & W_{2}^{2} & W_{3}^{2} & W_{4}^{2} & W_{5}^{2} & \vdots & \vdots & \vdots \\
\vdots & \vdots & \vdots & \vdots & \vdots & \vdots & \vdots & \vdots \\
0 & \cdots & W_{1}^{i} & W_{2}^{i} & W_{3}^{i} & W_{4}^{i} & W_{5}^{i} & \cdots \\
\vdots & \vdots & \vdots & \vdots & \vdots & \vdots & \vdots & \vdots \\
\vdots & \vdots & \vdots & W_{1}^{N-2} & W_{2}^{N-2} & W_{3}^{N-2} & \vdots W_{4}^{N-2} & W_{5}^{N-2} \\
0 & \cdots & \cdots & \cdots & W_{1}^{N-1} & W_{2}^{N-1} & W_{3}^{N-1} & W_{4}^{N-1} \\
0 & \cdots & \cdots & \cdots & \cdots & W_{1}^{N} & W_{2}^{N} & W_{3}^{N}
\end{array}\right]
$$

with coefficient matrix $\mathrm{B}=$

$\left[f\left(x_{0}\right)-A_{1} W_{1}^{0}-A_{2} W_{2}^{0}, f\left(x_{1}\right)-A_{2} W_{1}^{1}, f\left(x_{2}\right), \ldots, f\left(x_{N-2}\right), f\left(x_{N-1}\right)-A_{3} W_{5}^{N-1}, f\left(x_{N}\right)-A_{3} W_{4}^{N}-A_{4} W_{5}^{N}\right]^{t}$.

\section{Anatomy of Convergence}

This section is preserved for the exploration of the convergence of the quantic trigonometric Bspline collocation technique. We have assumed $C$ as a non-specific positive constant independent of $\delta, \varepsilon$ and $N$, which may capture different values at different points and $h=1 / N$ and $N=2^{m}$ to ensure at least one point in boundary layer.

Lemma 5.1 (Kumar and Kadalbajoo, 2012) If the functions $a(x), b(x), c(x), d(x)$ and $f(x)$ are sufficiently smooth and are independent of $\varepsilon$, then the solution y of (1) -(2) satisfies

$$
\left|y^{(k)}(x)\right| \leq C\left(1+\varepsilon^{-k} e^{-\frac{\alpha x}{\varepsilon}}\right), k=0,1,2 \ldots
$$


International Journal of Mathematical, Engineering and Management Sciences

Vol. 4, No. 6, 1471-1482, 2019

https://dx.doi.org/10.33889/IJMEMS.2019.4.6-116

For proof of this lemma, one can follow procedure similar to as given in Kellogg and Tsan (1978).

Lemma 5.2 Hall error estimation: If $f(x) \in C^{2}[0,1]$ and $y(x) \in C^{4}[0,1]$, then $\left\|D^{j}(y-Y)\right\| \leq$ $\lambda_{j}\left\|y^{4}\right\| h^{4-j}, j=0,1,2, \ldots$ where $\lambda_{j}$ are the constants (Hall, 1968).

Lemma 5.3 If $A$ is diagonally dominant by rows and $\alpha=\min _{\mathrm{i}}\left(\left|a_{i, i}\right|-\sum_{i \neq j}\left|a_{i, j}\right|\right)$. Then $\left\|A^{-1}\right\|_{\infty}<1 / \alpha($ Varah, 1975).

Theorem 5.1 Let $\mathrm{S}(\mathrm{x})$ be the approximation obtained by collocation method to the solution $\mathrm{y}(\mathrm{x})$ of boundary value problem (1)-(2). If $f \epsilon C^{2}[0,1]$, then the error estimate is given by $\sup _{\varepsilon} \max _{i}\left|y\left(x_{i}\right)-S\left(x_{i}\right)\right| \leq C N^{-1} \ln ^{3} N$, where $0 \leq i \leq N$ and $0<\varepsilon \leq 1$.

Proof: Consider $Y(x)$ be the unique spline interpolate to the solution $\mathrm{y}(\mathrm{x})$ of SPDDE given in (1)(2) given by (4) and the estimated error is given by $|y(x)-S(x)|$.

Now by using Hall error estimation as defined in Lemma 5.2 we get the following estimation:

$$
\begin{gathered}
\left|L y\left(x_{i}\right)-L Y\left(x_{i}\right)\right|=|-\varepsilon|\left|y^{\prime \prime \prime}\left(x_{i}\right)-Y^{\prime \prime \prime}\left(x_{i}\right)\right|+|a(x)-d(x)|\left|y^{\prime \prime}\left(x_{i}\right)-Y^{\prime \prime}\left(x_{i}\right)\right| \\
+|b(x)+d(x)|\left|y^{\prime}\left(x_{i}\right)-Y^{\prime}\left(x_{i}\right)\right|+|c(x)|\left|y\left(x_{i}\right)-Y\left(x_{i}\right)\right| \\
\leq\left(c_{\varepsilon} \lambda_{3} h+(\|a(x)\|+\|b(x)\|) \lambda_{2} h^{2}+(\|b(x)\|+\|d(x)\|) \lambda_{1} h^{3}+\|c(x)\| \lambda_{0} h^{4}\right)\left\|y^{4}\right\| .
\end{gathered}
$$

Using Lemma 5.1, we get

$$
\begin{gathered}
\left|L y\left(x_{i}\right)-L Y\left(x_{i}\right)\right| \leq\left(c_{\varepsilon} \lambda_{3} h+(\|a(x)\|+\|b(x)\|) \lambda_{2} h^{2}+(\|b(x)\|+\|d(x)\|) \lambda_{1} h^{3}\right. \\
\left.+\|c(x)\| \lambda_{0} h^{4}\right) C\left(1+\varepsilon^{-k} e^{-\alpha x / \varepsilon}\right)
\end{gathered}
$$

where $\varepsilon^{-1} \leq C \ln N$. Thus, $\left|L y\left(x_{i}\right)-L Y\left(x_{i}\right)\right| \leq C N^{-1} \ln ^{3} N$.

Therefore, we have $\left|L y\left(x_{i}\right)-L Y\left(x_{i}\right)\right|=\left|f\left(x_{i}\right)-L Y\left(x_{i}\right)\right| \leq C N^{-1} \ln ^{3} N$

Now consider the boundary value problem as $L Y(x)=\bar{f}\left(x_{i}\right)$ with conditions $Y\left(x_{0}\right)=$ $\phi(0), Y\left(x_{N}\right)=\gamma$.

$\mathrm{A} \bar{\alpha}=\bar{B}$ is a linear system of equations obtained from the above problem, which follows that

$\mathrm{A}(\alpha-\bar{\alpha})=B-\bar{B}$

where $B-\bar{B}=\left[f\left(x_{0}\right)-\bar{f}\left(x_{0}\right), f\left(x_{1}\right)-\bar{f}\left(x_{1}\right), \ldots, f\left(x_{N}\right)-\bar{f}\left(x_{N}\right)\right]^{t}$

By using (11), $\|B-\bar{B}\| \leq C N^{-1} \ln ^{3} N$

The matrix A is strictly diagonal dominant for sufficiently small values of $h$ and 
International Journal of Mathematical, Engineering and Management Sciences

Vol. 4, No. 6, 1471-1482, 2019

https://dx.doi.org/10.33889/IJMEMS.2019.4.6-116

$\left|a_{i, i}\right|-\left(\left|a_{i, i-1}\right|+\left|a_{i, i+1}\right|\right)=\left\{\begin{array}{c}F\left(x_{0}\right), \text { for first row } \\ L\left(x_{N}\right), \text { for last row } \\ M\left(x_{i}\right), \text { otherwise }\end{array}\right.$

where $F\left(x_{0}\right)=-\left(k_{13}+k_{14}\right) P\left(x_{0}\right)+\left(k_{10}-k_{9}-k_{8}\right) Q\left(x_{0}\right)-\left(k_{6}+k_{7}\right) R\left(x_{0}\right)+\left(k_{3}-k_{2}-\right.$ $\left.k_{1}\right) S\left(x_{0}\right)$,

$L\left(x_{N}\right)=-\left(k_{11}+k_{12}\right) P\left(x_{N}\right)+\left(k_{10}-k_{9}-k_{8}\right) Q\left(x_{N}\right)+\left(k_{5}-k_{4}\right) R\left(x_{N}\right)+\left(k_{6}-k_{2}-\right.$ $\left.k_{1}\right) S\left(x_{N}\right)$,

and $M\left(x_{i}\right)=-\varepsilon A^{\prime}+\left(a\left(x_{i}\right)-d\left(x_{i}\right)\right) A^{\prime \prime}-\left(b\left(x_{i}\right)+d\left(x_{i}\right)\right) A^{\prime \prime \prime}+c\left(x_{i}\right) A^{\prime \prime \prime \prime}$,

where $\quad A^{\prime}=k_{11}+k_{10}+k_{13}+k_{14}, A^{\prime \prime}=k_{10}-2 k_{8}-2 k_{9}, A^{\prime \prime \prime}=k_{4}+k_{5}+k_{6}+k_{7}, A^{\prime \prime \prime \prime}=$ $k_{3}-2 k_{1}-2 k_{2}$.

It is apparent, that $\mathrm{A}$ is strictly diagonally dominant. So, by using above and Lemma 5.3, it is concluded that

$\left\|A^{-1}\right\| \leq C$

Now combining (12), (13), and (14), $|\alpha-\bar{\alpha}| \leq C N^{-1} \ln ^{3} N, 0 \leq i \leq N$.

Similarly, estimating $\left|\alpha_{i}-\overline{\alpha_{l}}\right|$ from the boundary and assumed conditions as defined in Section 4 , we get,

$\max \left|\alpha_{i}-\bar{\alpha}_{l}\right| \leq C N^{-1} \ln ^{3} N$, for $-2 \leq i \leq N+2$

Now by using Lemma 5.1 and (15) to estimate $|S(x)-Y(x)|=\sum_{i=-2}^{N+2}\left(\alpha_{i}-\bar{\alpha}_{l}\right) T_{5, i}(x)$, we get $|S(x)-Y(x)| \leq C N^{-1} \ln ^{3} N$, which leads to result of theorem with triangleinequality.

$\sup (\varepsilon) \max (i)\left|y\left(x_{i}\right)-S\left(x_{i}\right)\right| \leq C N^{-1} \ln ^{3} N$, where $0 \leq i \leq N$ and $0<\varepsilon \leq 1$.

Hence the theorem is proved.

\section{Numerical Examples}

To validate the proposed scheme two examples are considered for the numerical simulation. As the exact solution of the equation is not known hence the double mesh principle is used to calculate the maximum absolute error and $\mathrm{D}^{\mathrm{N}}=\max \left|y_{i}^{N}-y_{2 i}^{2 N}\right|$ where $1 \leq i \leq N$.

Example 1: $\quad-\varepsilon y^{\prime \prime \prime}(x)+a(x) y^{\prime \prime}(x)+b(x) y^{\prime}(x)+c(x) y(x) d(x) 1 / 2 y^{\prime}(x-1)=0$,

$$
y(x)=1+x, x \in[-1,0], y^{\prime}(2)=2
$$

where $a_{1}=16, a_{2}=10, b(x)=0, c(x)=-1, d(x)=-1, f_{1}=1, f_{2}=-1$. 
International Journal of Mathematical, Engineering and Management Sciences

Vol. 4, No. 6, 1471-1482, 2019

https://dx.doi.org/10.33889/IJMEMS.2019.4.6-116

The maximum absolute error obtained for example 1 at different domain partitions is presented in

Table 1 and Figure 1 depicts the solution of Example 1 for $N=128$.

Table 1. Maximum absolute error of Example 1

\begin{tabular}{|c|c|c|c|c|c|c|c|}
\hline Epsilon & $\mathrm{N}=16$ & $\mathrm{~N}=32$ & $\mathrm{~N}=64$ & $\mathrm{~N}=128$ & $\mathrm{~N}=256$ & $\mathrm{~N}=512$ & $\mathrm{~N}=1024$ \\
\hline$\varepsilon=2^{-6}$ & $1.29 \mathrm{E}+00$ & $1.08 \mathrm{E}+00$ & $1.41 \mathrm{E}-01$ & $6.13 \mathrm{E}-03$ & $2.69 \mathrm{E}-04$ & $2.46 \mathrm{E}-02$ & $1.93 \mathrm{E}-01$ \\
\hline$\varepsilon=2^{-7}$ & $1.10 \mathrm{E}+00$ & $1.04 \mathrm{E}+00$ & $1.18 \mathrm{E}-01$ & $2.00 \mathrm{E}-03$ & $1.38 \mathrm{E}-06$ & $8.69 \mathrm{E}-09$ & $7.16 \mathrm{E}-08$ \\
\hline$\varepsilon=2^{-8}$ & $1.00 \mathrm{E}+00$ & $1.01 \mathrm{E}+00$ & $1.04 \mathrm{E}-01$ & $7.20 \mathrm{E}-04$ & $8.63 \mathrm{E}-07$ & $1.29 \mathrm{E}-11$ & $7.84 \mathrm{E}-18$ \\
\hline$\varepsilon=2^{-9}$ & $9.52 \mathrm{E}-01$ & $9.98 \mathrm{E}-01$ & $9.69 \mathrm{E}-02$ & $5.81 \mathrm{E}-04$ & $1.41 \mathrm{E}-07$ & $1.22 \mathrm{E}-13$ & $2.29 \mathrm{E}-23$ \\
\hline$\varepsilon=2^{-10}$ & $9.26 \mathrm{E}-01$ & $9.90 \mathrm{E}-01$ & $9.31 \mathrm{E}-02$ & $6.46 \mathrm{E}-04$ & $1.24 \mathrm{E}-07$ & $4.94 \mathrm{E}-15$ & $1.81 \mathrm{E}-27$ \\
\hline$\varepsilon=2^{-11}$ & $9.13 \mathrm{E}-01$ & $9.86 \mathrm{E}-01$ & $9.12 \mathrm{E}-02$ & $6.71 \mathrm{E}-04$ & $1.19 \mathrm{E}-07$ & $1.95 \mathrm{E}-15$ & $1.05 \mathrm{E}-30$ \\
\hline$\varepsilon=2^{-12}$ & $9.06 \mathrm{E}-01$ & $9.84 \mathrm{E}-01$ & $9.03 \mathrm{E}-02$ & $6.82 \mathrm{E}-04$ & $1.15 \mathrm{E}-07$ & $1.10 \mathrm{E}-15$ & $1.16 \mathrm{E}-30$ \\
\hline$\varepsilon=2^{-13}$ & $9.03 \mathrm{E}-01$ & $9.83 \mathrm{E}-01$ & $8.98 \mathrm{E}-02$ & $6.87 \mathrm{E}-04$ & $1.12 \mathrm{E}-07$ & $7.29 \mathrm{E}-16$ & $2.74 \mathrm{E}-31$ \\
\hline$\varepsilon=2^{-14}$ & $9.01 \mathrm{E}-01$ & $9.83 \mathrm{E}-01$ & $8.96 \mathrm{E}-02$ & $6.89 \mathrm{E}-04$ & $1.11 \mathrm{E}-07$ & $5.61 \mathrm{E}-16$ & $1.82 \mathrm{E}-31$ \\
\hline$\varepsilon=2^{-15}$ & $9.00 \mathrm{E}-01$ & $9.83 \mathrm{E}-01$ & $8.94 \mathrm{E}-02$ & $6.90 \mathrm{E}-04$ & $1.10 \mathrm{E}-07$ & $4.82 \mathrm{E}-16$ & $1.38 \mathrm{E}-31$ \\
\hline$\varepsilon=2^{-16}$ & $9.00 \mathrm{E}-01$ & $9.83 \mathrm{E}-01$ & $8.94 \mathrm{E}-02$ & $6.91 \mathrm{E}-04$ & $1.10 \mathrm{E}-07$ & $4.44 \mathrm{E}-16$ & $1.17 \mathrm{E}-31$ \\
\hline$\varepsilon=2^{-17}$ & $9.00 \mathrm{E}-01$ & $9.82 \mathrm{E}-01$ & $8.93 \mathrm{E}-02$ & $6.91 \mathrm{E}-04$ & $1.10 \mathrm{E}-07$ & $4.25 \mathrm{E}-16$ & $1.07 \mathrm{E}-31$ \\
\hline$\varepsilon=2^{-18}$ & $9.00 \mathrm{E}-01$ & $9.82 \mathrm{E}-01$ & $8.93 \mathrm{E}-02$ & $6.91 \mathrm{E}-04$ & $1.09 \mathrm{E}-07$ & $4.15 \mathrm{E}-16$ & $1.02 \mathrm{E}-31$ \\
\hline$\varepsilon=2^{-19}$ & $9.00 \mathrm{E}-01$ & $9.82 \mathrm{E}-01$ & $8.93 \mathrm{E}-02$ & $6.91 \mathrm{E}-04$ & $1.09 \mathrm{E}-07$ & $4.11 \mathrm{E}-16$ & $9.97 \mathrm{E}-32$ \\
\hline$\varepsilon=2^{-20}$ & $9.00 \mathrm{E}-01$ & $9.82 \mathrm{E}-01$ & $8.93 \mathrm{E}-02$ & $6.91 \mathrm{E}-04$ & $1.09 \mathrm{E}-07$ & $4.08 \mathrm{E}-16$ & $9.85 \mathrm{E}-32$ \\
\hline$\varepsilon=2^{-21}$ & $9.00 \mathrm{E}-01$ & $9.82 \mathrm{E}-01$ & $8.93 \mathrm{E}-02$ & $6.91 \mathrm{E}-04$ & $1.09 \mathrm{E}-07$ & $4.07 \mathrm{E}-16$ & $9.79 \mathrm{E}-32$ \\
\hline$\varepsilon=2^{-22}$ & $9.00 \mathrm{E}-01$ & $9.82 \mathrm{E}-01$ & 8.93E-02 & $6.91 \mathrm{E}-04$ & $1.09 \mathrm{E}-07$ & $4.07 \mathrm{E}-16$ & $9.76 \mathrm{E}-32$ \\
\hline$\varepsilon=2^{-23}$ & $9.00 \mathrm{E}-01$ & $9.82 \mathrm{E}-01$ & $8.93 \mathrm{E}-02$ & $6.91 \mathrm{E}-04$ & $1.09 \mathrm{E}-07$ & $4.06 \mathrm{E}-16$ & $9.75 \mathrm{E}-32$ \\
\hline
\end{tabular}

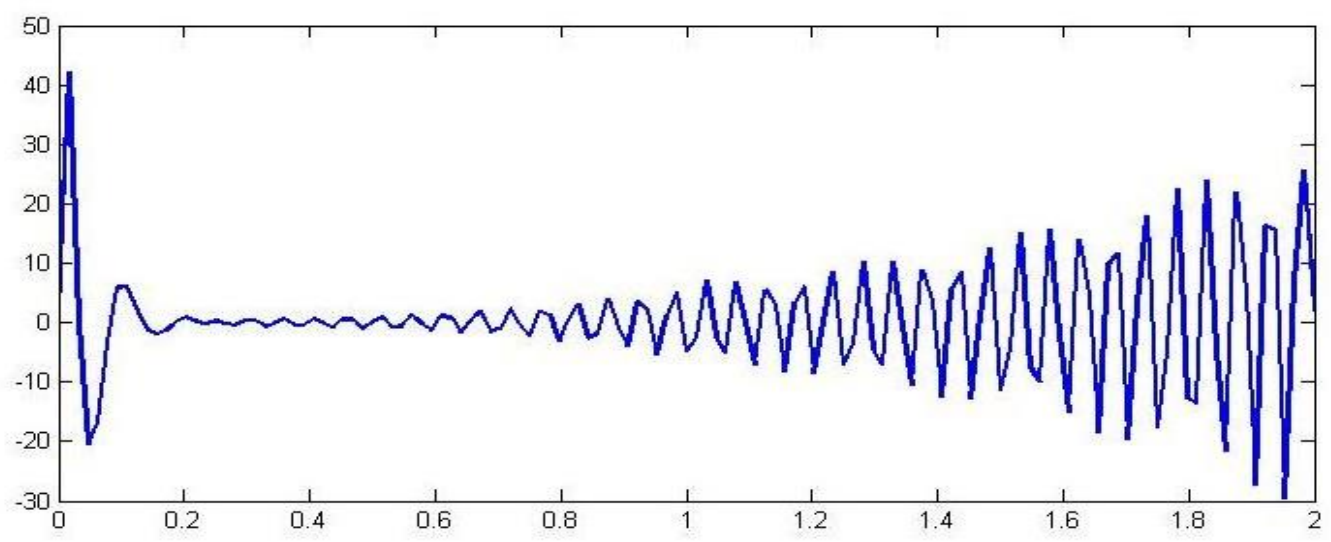

Figure 1. Graph of solution of Example 1 for $N=128$ and epsilon= $=.25$ 
International Journal of Mathematical, Engineering and Management Sciences

Vol. 4, No. 6, 1471-1482, 2019

https://dx.doi.org/10.33889/IJMEMS.2019.4.6-116

Example 2: $\quad-\varepsilon y^{\prime \prime \prime}(x)+a(x) y^{\prime \prime}(x)+b(x) y^{\prime}(x)+c(x) y(x) d(x) 1 / 2 y^{\prime}(x-1)=0$

$$
y(x)=1+x, x \in[-1,0], y^{\prime}(2)=2
$$

where $a_{1}=10+\exp \left(x^{2}\right), a_{2}=10+\exp (-\mathrm{x}), b(x)=3, c(x)=-1, d(x)=-1, f_{1}=1, f_{2}=$ -1 .

Table 2, demonstrates the maximum absolute error obtained for example 2 at different values of $N$. Figure 2 shows the solution of Example 2 for $N=128$.

Table 2. Maximum absolute error of Example 2

\begin{tabular}{|c|c|c|c|c|c|c|c|}
\hline Epsilon & $\mathrm{N}=16$ & $\mathrm{~N}=32$ & $\mathrm{~N}=64$ & $\mathrm{~N}=128$ & $\mathrm{~N}=256$ & $\mathrm{~N}=512$ & $\mathrm{~N}=1024$ \\
\hline$\varepsilon=2^{-6}$ & $3.37 \mathrm{E}+00$ & 7.46E-01 & $7.90 \mathrm{E}-02$ & $6.84 \mathrm{E}-03$ & 2.04E-04 & $1.67 \mathrm{E}-02$ & $2.48 \mathrm{E}-01$ \\
\hline$\varepsilon=2^{-7}$ & $3.13 \mathrm{E}+00$ & $5.87 \mathrm{E}-01$ & $8.32 \mathrm{E}-02$ & $2.23 \mathrm{E}-03$ & $5.57 \mathrm{E}-06$ & $5.61 \mathrm{E}-09$ & $9.54 \mathrm{E}-06$ \\
\hline$\varepsilon=2^{-8}$ & $3.01 \mathrm{E}+00$ & $5.16 \mathrm{E}-01$ & $8.74 \mathrm{E}-02$ & $9.33 \mathrm{E}-04$ & $7.23 \mathrm{E}-07$ & $1.02 \mathrm{E}-11$ & $4.45 \mathrm{E}-18$ \\
\hline$\varepsilon=2^{-9}$ & $2.95 \mathrm{E}+00$ & $4.83 \mathrm{E}-01$ & $8.79 \mathrm{E}-02$ & $4.20 \mathrm{E}-04$ & 7.41E-08 & $1.49 \mathrm{E}-13$ & $2.19 \mathrm{E}-23$ \\
\hline$\varepsilon=2^{-10}$ & $2.92 \mathrm{E}+00$ & $4.67 \mathrm{E}-01$ & $8.78 \mathrm{E}-02$ & $2.06 \mathrm{E}-04$ & $4.57 \mathrm{E}-08$ & $1.67 \mathrm{E}-15$ & $1.34 \mathrm{E}-27$ \\
\hline$\varepsilon=2^{-11}$ & $2.91 \mathrm{E}+00$ & $4.59 \mathrm{E}-01$ & $8.77 \mathrm{E}-02$ & $1.91 \mathrm{E}-04$ & $6.42 \mathrm{E}-08$ & $2.49 \mathrm{E}-15$ & $1.68 \mathrm{E}-29$ \\
\hline$\varepsilon=2^{-12}$ & $2.90 \mathrm{E}+00$ & $4.55 \mathrm{E}-01$ & $8.76 \mathrm{E}-02$ & $1.97 \mathrm{E}-04$ & $7.28 \mathrm{E}-08$ & $1.85 \mathrm{E}-15$ & $3.69 \mathrm{E}-31$ \\
\hline$\varepsilon=2^{-13}$ & $2.90 \mathrm{E}+00$ & $4.53 \mathrm{E}-01$ & $8.76 \mathrm{E}-02$ & $1.98 \mathrm{E}-04$ & 7.57E-08 & $1.42 \mathrm{E}-15$ & $2.65 \mathrm{E}-31$ \\
\hline$\varepsilon=2^{-14}$ & $2.90 \mathrm{E}+00$ & $4.52 \mathrm{E}-01$ & $8.75 \mathrm{E}-02$ & 1.99E-04 & 7.69E-08 & $1.21 \mathrm{E}-15$ & $2.34 \mathrm{E}-31$ \\
\hline$\varepsilon=2^{-15}$ & $2.89 \mathrm{E}+00$ & $4.52 \mathrm{E}-01$ & $8.75 \mathrm{E}-02$ & $2.00 \mathrm{E}-04$ & 7.74E-08 & $1.10 \mathrm{E}-15$ & $1.96 \mathrm{E}-31$ \\
\hline$\varepsilon=2^{-16}$ & $2.89 \mathrm{E}+00$ & $4.51 \mathrm{E}-01$ & $8.75 \mathrm{E}-02$ & $2.00 \mathrm{E}-04$ & 7.76E-08 & $1.05 \mathrm{E}-15$ & $1.74 \mathrm{E}-31$ \\
\hline$\varepsilon=2^{-17}$ & $2.89 \mathrm{E}+00$ & $4.51 \mathrm{E}-01$ & $8.75 \mathrm{E}-02$ & $2.00 \mathrm{E}-04$ & $7.78 \mathrm{E}-08$ & $1.02 \mathrm{E}-15$ & $1.63 \mathrm{E}-31$ \\
\hline$\varepsilon=2^{-18}$ & $2.89 \mathrm{E}+00$ & $4.51 \mathrm{E}-01$ & $8.75 \mathrm{E}-02$ & $2.00 \mathrm{E}-04$ & $7.78 \mathrm{E}-08$ & $1.01 \mathrm{E}-15$ & $1.57 \mathrm{E}-31$ \\
\hline$\varepsilon=2^{-19}$ & $2.89 \mathrm{E}+00$ & $4.51 \mathrm{E}-01$ & $8.75 \mathrm{E}-02$ & $2.00 \mathrm{E}-04$ & $7.78 \mathrm{E}-08$ & $1.00 \mathrm{E}-15$ & $1.54 \mathrm{E}-31$ \\
\hline$\varepsilon=2^{-20}$ & $2.89 \mathrm{E}+00$ & $4.51 \mathrm{E}-01$ & $8.75 \mathrm{E}-02$ & $2.00 \mathrm{E}-04$ & 7.79E-08 & $1.00 \mathrm{E}-15$ & $1.53 \mathrm{E}-31$ \\
\hline$\varepsilon=2^{-21}$ & $2.89 \mathrm{E}+00$ & $4.51 \mathrm{E}-01$ & $8.75 \mathrm{E}-02$ & $2.00 \mathrm{E}-04$ & 7.79E-08 & $1.00 \mathrm{E}-15$ & $1.52 \mathrm{E}-31$ \\
\hline$\varepsilon=2^{-22}$ & $2.89 \mathrm{E}+00$ & $4.51 \mathrm{E}-01$ & $8.75 \mathrm{E}-02$ & $2.00 \mathrm{E}-04$ & 7.79E-08 & $9.99 \mathrm{E}-16$ & $1.52 \mathrm{E}-31$ \\
\hline$\varepsilon=2^{-23}$ & $2.89 \mathrm{E}+00$ & $4.51 \mathrm{E}-01$ & $8.75 \mathrm{E}-02$ & $2.00 \mathrm{E}-04$ & $7.79 \mathrm{E}-08$ & $9.99 \mathrm{E}-16$ & $1.52 \mathrm{E}-31$ \\
\hline
\end{tabular}


International Journal of Mathematical, Engineering and Management Sciences

Vol. 4, No. 6, 1471-1482, 2019

https://dx.doi.org/10.33889/IJMEMS.2019.4.6-116

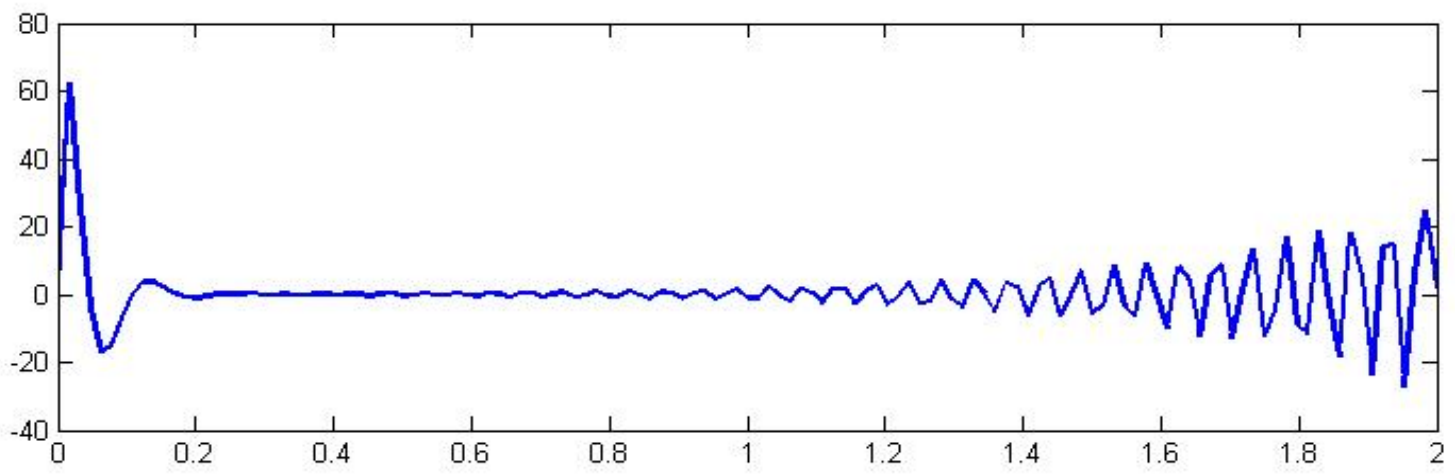

Figure 2. Graph of solution of Example 2 for $\mathrm{N}=128$ and epsilon= 0.25

\section{Conclusion}

A class of third order SPDDE for ordinary delay differential equations with large delay is considered with discontinuous convection-diffusion coefficient and source term. In section 3 existence of the solution of the problem is shown. With the uniform partition of the domain, the solution is approximated by quintic trigonometric B-spline basis by collocation technique. Discussion of convergence is carried out by the Hall theorem and the order of convergence of the method presented in this paper is of the almost first order. It can be concluded from the numerical results that maximum absolute error declines as $N$ increases. Hence the presented scheme is efficient to simulate the considered SPDDE and other related types of differential equations.

\section{Conflict of Interest}

The authors confirm that this article contents have no conflict of interest.

\section{Acknowledgement}

The authors would like to express their sincere thanks to the editor and referee for their valuable suggestions towards the improvement of the paper.

\section{References}

Andargie, A., \& Reddy, Y. N. (2013). Parameter fitted scheme for singularly perturbed delay differential equations. International Journal of Applied Science and Engineering, 11(4), 361-373.

Cimen, E. (2017). A priori estimates for solution of singularly perturbed boundary value problem with delay in convection term. Journal of Mathematical Analysis, 8(1), 202-211.

Hall, C.A. (1968). On error bounds for spline interpolation. Journal of Approximation Theory, 1(2), 209218.

Kellogg, R.B., \& Tsan, A. (1978). Analysis of some difference approximations for a singular perturbation problem without turning points. Mathematics of Computation, 32(144), 1025-1039. 
International Journal of Mathematical, Engineering and Management Sciences

Vol. 4, No. 6, 1471-1482, 2019

https://dx.doi.org/10.33889/IJMEMS.2019.4.6-116

Kumar, D., \& Kadalbajoo, M.K. (2012). Numerical treatment of singularly perturbed delay differential equations using B-Spline collocation method on Shishkin mesh. Journal of Numerical Analysis, Industrial and Applied Mathematics, 7(3-4), 73-90.

Longtin, A., \& Milton, J.G. (1988). Complex oscillations in the human pupil light reflex with "mixed" and delayed feedback. Mathematical Biosciences, 90(1-2), 183-199.

Nelson, P.W., \& Perelson, A.S. (2002). Mathematical analysis of delay differential equation models of HIV-1 infection. Mathematical Biosciences, 179(1), 73-94.

Nicaise, S., \& Xenophontos, C. (2013). Robust approximation of singularly perturbed delay differential equations by the hp finite element method. Computational Methods in Applied Mathematics, 13(1), 2137.

Rihan, F.A. (2013). Delay differential equations in biosciences: parameter estimation and sensitivity analysis. In Recent Advances in Applied Mathematics and Computational Methods: Proceedings of the 2013 International Conference on Applied Mathematics and Computational Methods (Venice, Italy September 2013) (pp. 50-58).

Stein, R.B. (1965). A theoretical analysis of neuronal variability. Biophysical Journal, 5(2), 173-194.

Stein, R.B. (1967). Some models of neuronal variability. Biophysical Journal, 7(1), 37-68.

Subburayan, V., \& Mahendran, R. (2018). An $\varepsilon$-uniform numerical method for third order singularly perturbed delay differential equations with discontinuous convection coefficient and source term. Applied Mathematics and Computation, 331, 404-415.

Swamy, D.K., Phaneendra, K., Babu, A.B., \& Reddy, Y.N. (2015). Computational method for singularly perturbed delay differential equations with twin layers or oscillatory behaviour. Ain Shams Engineering Journal, 6(1), 391-398.

Varah, J.M. (1975). A lower bound for the smallest singular value of a matrix. Linear Algebra and its Applications, 11(1), 3-5.

Wilkie, K P., \& Hahnfeldt, P. (2013). Mathematical models of immune-induced cancer dormancy and the emergence of immune evasion. Interface Focus, 3(4), 20130010.

Zakaria, N.F., Hassan, N.A., Hamid, N.N.A., Majid, A.A., \& Ismail, A.I.M. (2017, April). Solving boussinesq equation using quintic B-spline and quintic trigonometric B-spline interpolation methods. In AIP Conference Proceedings 1830(1), p. 020041. AIP Publishing.

Zhang, Y., Jie, Y., \& Meng, X. (2016). The modelling and control of a singular biological economic system in a polluted environment. Discrete Dynamics in Nature and Society, 2016, Article ID 3925386, 7 pages. 